\title{
Territorios insulares y unidades de soporte vital avanzado
}

\section{Insular territories and advanced life support units}

\author{
D. Arroyo-Morales, R. Delgado-Lorenzo, A. Díaz-Cabrera, M. Hernández-Hernández
}

\section{Sr. Director:}

El trabajo publicado por Ballesteros ${ }^{1}$, sobre el número y la distribución territorial y poblacional de las ambulancias destinadas a la atención a las urgencias sanitarias, presenta una metodología adecuada en su planteamiento y novedosa por su simplicidad, y contribuye a conocer la situación de los sistemas de emergencias médicas (SEM) en España, de forma que el estudio es, a partir de su publicación, un referente necesario en la bibliografía de las emergencias sanitarias en España.

Sin embargo echamos de menos en la discusión algún comentario sobre los territorios insulares de Baleares y Canarias. En primer lugar porque al ser un territorio discontinuo, las unidades de soporte vital básico y avanzado pueden parecer "a priori" superiores a la media nacional, cuando precisamente la fragmentación del territorio hace necesario disponer de recursos en forma diferente al territorio continental. Además, aunque el trabajo se centra en los medios de transporte terrestres, también en la discusión deben tenerse en cuenta los recursos aéreos medicalizados (un avión y dos helicópteros en el caso de Canarias) ${ }^{2}$, los cuales reducen las isocronas de actuación entre islas, tanto en operaciones primarias como en los traslados interhospitalarios. Algo similar puede ocurrir en algunas comunidades autónomas peninsulares con una gran superficie ${ }^{3,4}$.

Otro aspecto a considerar en la comparación del número y distribución de las ambulancias entre comunidades autónomas, es la población flotante de determinadas regiones, fundamentalmente turística. En el caso de Canarias, frente a una población censada de 2,1 millones, se registró en 2011 una cifra de 12 millones de visitantes, factor a tener en cuenta para el cálculo de las ambulancias necesarias para la asistencia de la población real existente. Probablemente cálculos similares deben realizarse en ciudades con gran afluencia de visitantes como Madrid, Barcelona y zonas turísticas como Levante o las Islas Baleares.

Así pues, estas matizaciones creemos que ayudan a contextualizar mejor los datos de densidad e isocronas de ambulancias que "a priori" podrían parecer no justificados, como esboza Ballesteros, en función de la idiosincrasia demográfica de cada región y también en la heterogeneidad
Facultad de Ciencias de la Salud. Universidad Europea de Canarias. Tenerife.
Recepción: 16 de enero de 2013

Aceptación definitiva: 21 de enero de 2013

\section{Correspondencia:}

Roberto Delgado Lorenzo

Universidad Europea de Canarias

c/ Inocencio García, 1

38300 La Orotava

Tenerife

E-mail.robertodl86@gmail.com 
de los SEM, probablemente necesaria, por las dos razones anteriormente expuestas, entre otras. Sin esta contextualización se correría el riesgo de que algún gestor sanitario utilizase los datos de forma errónea ${ }^{5}$, máxime en la actual situación de racionalización de recursos públicos.

\section{BIBLIOGRAFIA}

1. Ballesteros S. Unidades de soporte vital básico y avanzado en España: análisis de la situación actual. An Sist Sanit Nav 2012; 35: 219-228.

2. Lubillo S. Burillo-Putze G, Alonso E, Herranz I, Gómez A, Gómez N. Helicopter emergency medical service in Canary Islands, Spain. Eur J Emerg Med 2000; 7: 55-59.
3. Mateos Rodríguez A, Navalpotro Pascual JM, Ortea I, Moreno R, Barba Alonso C, Martín MalDONADo ME et al. Traslado en helicóptero de donantes tras muerte cardiaca. Emergencias 2011; 23: 372-374.

4. Cardenete Reyes C, Polo Portes CE, Téllez GALÁN G. Escala de valoración del riesgo del transporte interhospitalario de pacientes críticos: su aplicación en el Servicio de Urgencias Médicas de Madrid (SUMMA (SUMMA 112). Emergencias 2011; 23: 35-38.

5. Moreno Millán E, Villegas del Ojo J, García Tordecilla JM, Lea Pereira MC, Prieto Valderrey F. Acerca de la aplicación de una escala de evaluación del riesgo para el transporte interhospitalario: necesidad de adaptación a la situación actual y rediseño de componentes. Emergencias 2012; 27: 74-80. 